\title{
Neuroprotective and Anti-Microglial Activation Effects of Tocotrienols in Brains of Lipopolysaccharide-Induced Inflammatory Model Mice
}

\author{
Satoshi Okuyama ${ }^{1,2,3, *}$, Masafumi Matsuda ${ }^{1}$, Yuna Okusako ${ }^{1}$, Sanae Miyauchi ${ }^{1}$, Toshiki Omasa ${ }^{2}$, \\ Akiho Ozawa ${ }^{4}$, Masato Abe ${ }^{3,4,5}$, Takashi Yaeno ${ }^{3,6}$, Takuya Araki ${ }^{3,7}$, Atsushi Sawamoto ${ }^{1,2}$, \\ Mitsunari Nakajima ${ }^{1,2}$ and Yoshiko Furukawa ${ }^{1,2}$ (iD)
}

check for updates

Citation: Okuyama, S.; Matsuda, M.; Okusako, Y.; Miyauchi, S.; Omasa, T.; Ozawa, A.; Abe, M.; Yaeno, T.; Araki, T.; Sawamoto, A.; et al.

Neuroprotective and Anti-Microglial Activation Effects of Tocotrienols in Brains of LipopolysaccharideInduced Inflammatory Model Mice. Neuroglia 2021, 2, 89-97. https:// doi.org/10.3390/neuroglia2010009

Academic Editor: James St John

Received: 18 November 2021 Accepted: 15 December 2021 Published: 16 December 2021

Publisher's Note: MDPI stays neutral with regard to jurisdictional claims in published maps and institutional affiliations.

Copyright: (c) 2021 by the authors Licensee MDPI, Basel, Switzerland. This article is an open access article distributed under the terms and conditions of the Creative Commons Attribution (CC BY) license (https:// creativecommons.org/licenses/by/ $4.0 /)$.
1 Department of Pharmaceutical Pharmacology, College of Pharmaceutical Sciences, Matsuyama University, Matsuyama 790-8578, Japan; mu.yakuri.017@gmail.com (M.M.); 16170213@g.matsuyama-u.ac.jp (Y.O.); 16160868@g.matsuyama-u.ac.jp (S.M.); asawamot@g.matsuyama-u.ac.jp (A.S.); mnakajim@g.matsuyama-u.ac.jp (M.N.); furukawa@g.matsuyama-u.ac.jp (Y.F.)

2 Department of Pharmaceutical Pharmacology, Graduate School of Clinical Pharmacy, Matsuyama University, Matsuyama 790-8578, Japan; 46210023@g.matsuyama-u.ac.jp

3 Center for Development of Hulless Barley, Graduate School of Agriculture, Ehime University, Matsuyama 790-8566, Japan; abe.masato.si@ehime-u.ac.jp (M.A.); yaeno@agr.ehime-u.ac.jp (T.Y.); araki@agr.ehime-u.ac.jp (T.A.)

4 Laboratory of Natural Products Organic Chemistry, Department of Bioscience, Faculty of Agriculture, Ehime University, Matsuyama 790-8566, Japan; f612006a@mails.cc.ehime-u.ac.jp

5 Laboratory of Natural Products Organic Chemistry, Department of Bioscience, Graduate School of Agriculture, Ehime University, Matsuyama 790-8566, Japan

6 Laboratory of Plant Pathology, Department of Food Production Science, Graduate School of Agriculture, Ehime University, Matsuyama 790-8566, Japan

7 Laboratory of Crop Science, Department of Food Production Science, Graduate School of Agriculture, Ehime University, Matsuyama 790-8566, Japan

* Correspondence: sokuyama@g.matsuyama-u.ac.jp; Tel.: +81-89-925-7111

Abstract: Inflammation is the cause and/or result of many diseases in peripheral tissues and the central nervous system. Recent findings suggested that inflammation in peripheral tissue induces an inflammatory response in the brain that activates glial cells, which, in turn, induce neuronal cell dysfunction. Therefore, anti-inflammatory compounds are important for the suppression of chronic inflammation and prevention of disease. The present study revealed microglial activation in the hippocampus of the brain two days after the peripheral administration of lipopolysaccharide (LPS). Furthermore, the expression of the synaptic vesicle membrane protein, synaptophysin, in the CA3 stratum lucidum of the hippocampus was down-regulated 7 days after the LPS injection. The administration of tocotrienols, a type of vitamin E, significantly attenuated these changes in the hippocampus. Collectively, the present results demonstrated the spread of peripheral inflammatory responses to the brain, in which glial activation and neuronal dysfunction were induced, while tocotrienols exerted anti-inflammatory effects and protected neurons from damage.

Keywords: tocotrienols; brain; anti-inflammation; microglia

\section{Introduction}

Chronic inflammation has been implicated in the pathogenesis of many diseases, including cancer, rheumatic disease, diabetes, hypertension, and obesity. Inflammation in the brain occurs due to traumatic brain injury as well as cerebrovascular disease and neurodegenerative disorders, such as Alzheimer's disease and Parkinson's disease. Recent findings reported that chronic peripheral inflammation induced brain inflammation [1-3]. The activation of immunocompetent cells in the blood, including monocytes, neutrophils, and lymphocytes, by chronic inflammation in peripheral tissue has been shown to stimulate 
inflammatory responses by vascular endothelial cells, which cross the blood-brain barrier (BBB) and activate glial cells that induce aseptic neuronal cell damage [4,5]. Lipopolysaccharide (LPS), an endotoxin of the outer membrane of Gram-negative bacteria, is often used as an inflammation-induced model both in vitro and vivo. It stimulates Toll-like receptor 4 (TLR4) on the cell surface of immunocompetent cells, which release inflammatory substances, such as cytokines. The effects of many anti-inflammatory agents from natural sources have been examined and reported using this LPS-induced inflammation animal model [6-8].

Vitamin $\mathrm{E}$ is divided into two types, tocopherols and tocotrienols, with the latter comprising four subtypes: $\alpha, \beta, \gamma$, and $\delta$. Tocotrienols are present in palm oil, rice bran, barley, wheat germ, and rye [9]. In vitro and in vivo studies on tocotrienols demonstrated their suppressive effects on macrophage activation and the release of cytokines [10,11]; however, few studies have investigated their effects on brain function. Tocotrienols have been shown to exert inhibitory effects on microglia in addition to neuroprotective effects [12-14], and unsaturated chains in their structure have been suggested to enable their permeation into the brain [15]. However, the relationship between tocotrienols and inflammation in the brain remains unclear. Therefore, we herein examined the effects of tocotrienols in a mouse model of LPS-induced inflammation.

\section{Materials and Methods}

\subsection{Sample Preparation and LPS Treatment}

A tocotrienol mixture (in olive oil) was obtained from DHC Corporation (\#2204, DHC, Tokyo, Japan) and prepared for peroral (p.o.) administration at a sample concentration of $200 \mathrm{mg} / 10 \mathrm{~mL} / \mathrm{kg}$ body weight (BW) to the tocotrienol group. Control and LPS groups received vehicle solution of olive oil (10 mL/kg BW; \#150-00276, Wako Pure Chemical, Tokyo, Japan).

LPS (L7261, Sigma-Aldrich, St. Louis, MO, USA) was dissolved in saline. On Day 8 after the administration of each sample, LPS solution was intraperitoneally (i.p.) injected $(2 \mathrm{mg} / 10 \mathrm{~mL} / \mathrm{kg} \mathrm{BW})$ into mice in the LPS-treated and tocotrienol groups. Vehicle $(10 \mathrm{~mL} / \mathrm{kg}$ BW of saline) was i.p. administered to the non-treated control group.

\subsection{Experimental Schedule}

Four-week-old male ICR mice were purchased from Japan SLC (Hamamatsu, Japan). Mice were kept in an animal room at $23 \pm 1{ }^{\circ} \mathrm{C}$ on a 12-h light/dark cycle (lights on 8:00-20:00). Tap water and the experimental stock diet were freely available during the experimental period. All animal experiments were performed in accordance with the Guidelines for Animal Experimentation, approved by the Animal Care and Use Committee, and implemented using the approved protocol (\#17-011).

After one week of habituation, mice (age 5 weeks) were randomly divided into the following three groups ( $n=8$, respectively): vehicle (olive oil) administration in the vehicle (saline)-injected group (CON), vehicle administration in the LPS ( $2 \mathrm{mg} / \mathrm{kg} \mathrm{BW})$-injected group (LPS), and tocotrienol mixture $(200 \mathrm{mg} / \mathrm{kg} \mathrm{BW})$ administration in the LPS-injected group (LPS + T3). Vehicle or tocotrienol samples were administrated once a day from Days 1 to 9 (Experiment 1) or 14 (Experiment 2) and mice were then injected with either vehicle or LPS on Day 8 (Experiments 1 and 2). Mice were sacrificed on Day 10 in Experiment 1 and on Day 15 in Experiment 2. During the dissection procedure, mice were transcardially perfused with heparinized ice-cold phosphate-buffered saline through the left ventricle, and the brains were removed. The hippocampus isolated from one hemisphere of the brain was stored at $-80{ }^{\circ} \mathrm{C}$ and that from the other hemisphere was postfixed in $4 \%$ paraformaldehyde.

\subsection{Immunohistochemical Staining}

Thirty-micrometer-thick sagittal sections of frozen brains were cut using a cryostat (CM3050S; Leica Microsystems, Wetzlar, Germany). Brain sections were immunostained 
using a rabbit polyclonal antibody against Iba1 (1:1000; \#019-19741, Wako Pure Chemical) as the primary antibody to stain microglia/macrophages. The secondary antibody used was an EnVision-plus system HRP-labeled polymer (K4003, Dako, Glostrup, Denmark). Immunoreactivity was developed and visualized by DAB substrate (SK-4100, Vector Laboratories, Burlingame, CA, USA). ImageJ software (NIH, Bethesda, MD, USA) was used for the quantification of Iba1-positive signals.

\subsection{Immunofluorescence Staining}

Thirty-micrometer-thick sagittal sections were incubated with goat anti-synaptophysin (1:1000; \#5768, Sigma-Aldrich, St. Louis, MO, USA) as the primary antibody. Alexa Fluor 488 goat anti-mouse IgG (H + L) (1:300; A11029, Invitrogen, Carlsbad, CA, USA) was used as the secondary antibody. Sections were covered with mounting medium with DAPI (Vectashield; Vector Laboratories, Burlingame, CA, USA), and synaptophysin-positive signals in the cornu ammonis 3 (CA3) region of the hippocampus in each section were captured using a confocal fluorescence microscopy system (LSM800, Zeiss, Oberkochen, Germany). Captured signals were quantified using ImageJ software (1.48v, NIH, Bethesda, MD, USA).

\subsection{Western Blotting}

Hippocampal tissues were subjected to a Western blot analysis. Tissues were homogenized in 10 volumes $(w / v)$ of RIPA buffer [ $20 \mathrm{mM}$ Tris- $\mathrm{HCl}, \mathrm{pH} 7.5,150 \mathrm{mM} \mathrm{NaCl}, 0.1 \%$ SDS, $1 \%$ sodium deoxycholate, $1 \% \mathrm{NP}-40,2 \mathrm{mM}$ EDTA, and a protease inhibitor cocktail (Roche, Mannheim, Germany)]. Lysates were centrifuged at $20,000 \times \mathrm{g}$ at $4{ }^{\circ} \mathrm{C}$ for $30 \mathrm{~min}$ and supernatant solutions were collected as the protein extract. Equal amounts of protein $(25 \mu \mathrm{g})$ were separated on $8 \%$ SDS polyacrylamide gels and electroblotted onto an ImmunoBlot PVDF Membrane (Bio-Rad, Hercules, CA, USA). The primary antibody was against high mobility group box-1 protein (HMGB1, 1:10,000; ab79823, Abcam, Cambridge, UK) or actin (1:10,000; A2066, Sigma-Aldrich), and the secondary antibody was horseradish peroxidase-linked anti-rabbit IgG (1:10,000; \#7074, Cell Signaling, Woburn, MA, USA). Immunoreactive bands were visualized by ECL-prime (GE Healthcare, Little Chalfont, UK), and band intensities were measured using a ChemiDoc Touch MP imaging system and ImageLab software (v6.0.0, Bio-Rad).

\subsection{HPLC Analysis}

Tocotrienols were extracted twice with ethyl acetate/water. The organic layer was dried over $\mathrm{MgSO} 4$ and evaporated under reduced pressure. The residue was dissolved in n-hexane $(10 \mathrm{~mL})$ and the solution was diluted (1:599) in n-hexane for an HPLC analysis (Shimadzu 10Avp system; Shimadzu, Kyoto, Japan). The chromatographic separation of tocotrienols was performed using the normal phase method described in previous studies [16-18] with slight modifications. A $100 \times 4.6 \mathrm{~mm}$ i.d., $3 \mu \mathrm{m}$ particle size, Unison UK Silica column (Imtakt, Kyoto, Japan) was used at $40{ }^{\circ} \mathrm{C}$ in a column oven (CTO-10Avp). The mobile phase was n-hexane/1,4-dioxane $(99: 1 \mathrm{v} / \mathrm{v})$ at a flow late of $2.0 \mathrm{~mL} / \mathrm{min}$ by LC-10Avp. The mobile phase was previously degassed by sonication for $30 \mathrm{~min}$. The detection of Abs296 in all peaks was performed with SPD-10Avp.

\subsection{Statistical Analysis}

Data for individual groups are expressed as means \pm SEM. Data for bar graphs were analyzed using a $t$-test between the CON and LPS groups, or LPS and LPS + T3 groups, and a two-way ANOVA followed by Dunnett's multiple comparison test was applied for time-course graphs using Prism 6 (GraphPad Software, La Jolla, CA, USA). A value of $p<0.05$ was considered to be significant. 


\section{Results}

\subsection{BW Changes (Experiments 1 and 2)}

As shown in Figure 1, BW increased in all three groups, and no significant differences were observed between the vehicle and T3 treatments in the 7 days before the LPS injection. However, significant decreases in BW were observed after the LPS injection in both the LPS and LPS + T3 groups on Day 10. BW was slightly lower in the LPS groups than in the CON group $(p=0.1)$, whereas no significant differences were noted between the LPS and LPS + T3 groups (Exp 1; Figure 1a). BW increased in both the LPS and LPS + T3 groups on Day 15 (Exp 2; Figure 1b).

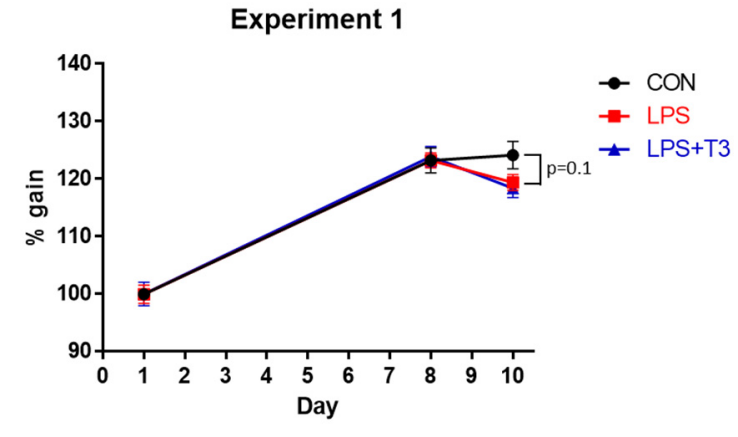

(a)

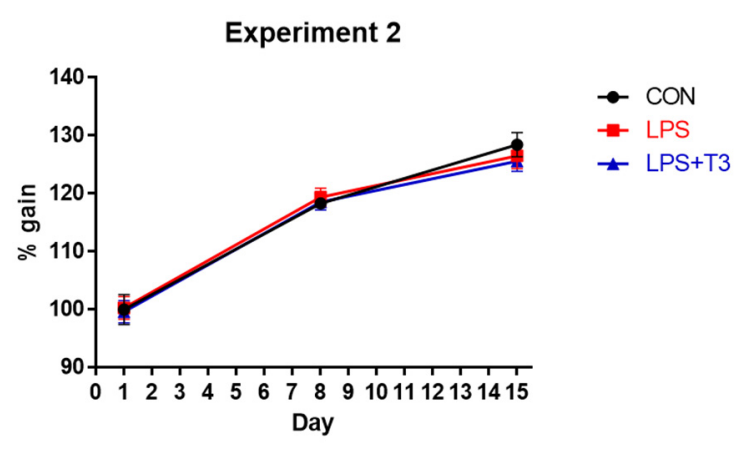

(b)

Figure 1. Body weight changes in Experiments 1 (a) and 2 (b). Values are means \pm SEM ( $n=8$, each group). Differences were observed between CON vs. LPS in timepoint comparisons on Day 10 in Experiment 1 ( $p=0.1$; a two-way ANOVA followed by Dunnett's multiple comparison test).

\subsection{Suppression of Microglial Activation in the Hippocampus (Experiment 1)}

With the systemic administration of LPS-activated microglia in the hippocampus, morphologically, many ameboid-type microglia were observed in the LPS group, whereas ameboid-type microglia and ramified-type microglia decreased and increased, respectively, in the LPS + T3 group (Figure 2a). The number of Iba1-positive cells was significantly higher in the LPS group than in the CON group, and this increase was significantly suppressed in the LPS + T3 group. Similar results were obtained for the integrated density of Iba1-positive cell signals (Figure 2b).

CON

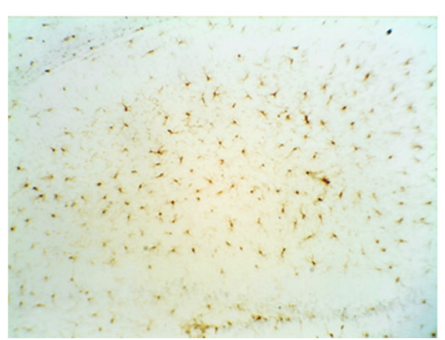

Figure 2. Cont
LPS

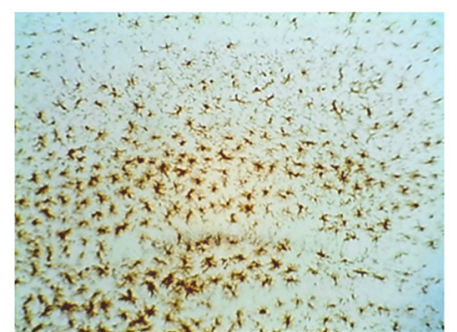

(a)
LPS + T3

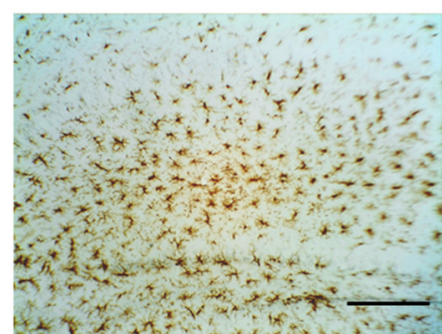



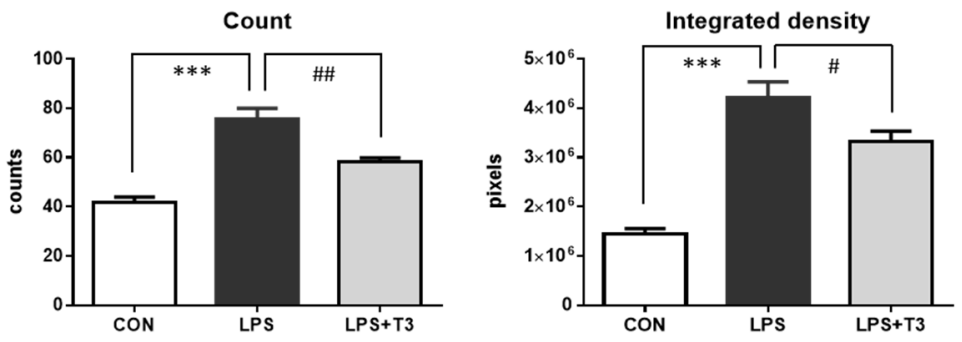

(b)

Figure 2. Effects of tocotrienols on Iba1 immunoreactivity in the hippocampus on Day 10 in Experiment 1. (a) Sagittal sections were stained with the anti-Iba1 antibody. The scale bar shows $50 \mu \mathrm{m}$. (b) Quantitative analysis of Iba1-positive cell numbers and the integrated density of signals using ImageJ software. Values are means $\pm \operatorname{SEM}(n=12$, each group). Symbols show significant differences between CON vs. LPS (*** $p<0.001$; $t$-test) and LPS vs. LPS + T3 ${ }^{*} p<0.05,{ }^{* \#} p<0.01$; $t$-test).

\subsection{Western Blot Analysis of Inflammation Markers in the Hippocampus (Experiment 1)}

The activation of microglia is an inflammatory response in the brain. HMGB1 is one of the damage-associated molecular patterns (DAMPs) and a marker of delayed-onset inflammation. The expression of HMGB1 in the hippocampus was significantly higher in the LPS group than in the CON group, while the LPS + T3 group did not show any significant suppression in this timepoint analysis (Figure 3).

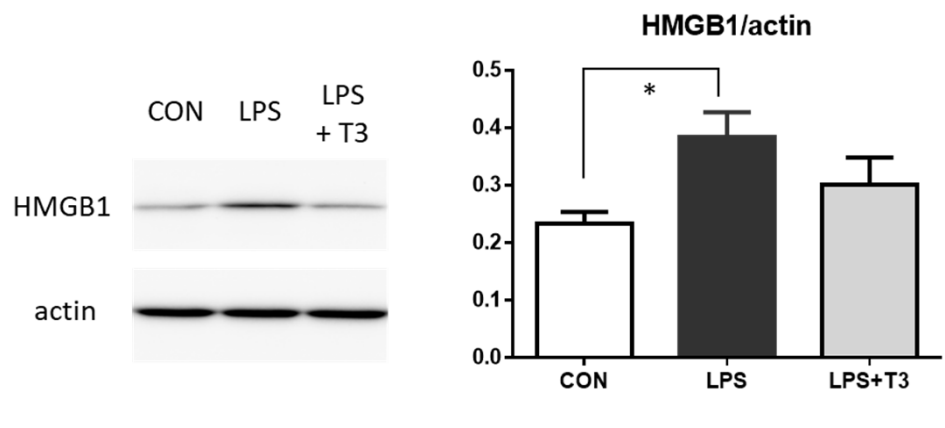

(a)

(b)

Figure 3. Effects of tocotrienols on the expression of HMGB1 in the hippocampus in Experiment 1. (a) Western blot analysis with the anti-HMGB1 antibody (27 kDa) and anti-actin antibody (42 kDa).

(b) Quantification of band intensities using ImageJ software. Values are means \pm SEM ( $n=6$, each group). The symbol shows a significant difference for CON vs. LPS (* $p<0.05 ;$-test).

\subsection{Suppression of Neuronal Dysfunction in the Hippocampus (Experiment 2)}

The CA3 stratum lucidum, at which the projections of hippocampal mossy fibers form synapses with CA3 pyramidal cells, is an important area for memory formation. Synaptophysin is a marker for synaptic vesicle membrane proteins. Anti-synaptophysin antibody-positive signals were strongly detected in the hippocampal CA3 region in the CON group, but were weaker in the LPS group (Figure $4 a, b,{ }^{* *} p<0.001$ ). Synaptic conditions were more stably maintained in the LPS + T3 group than in the LPS group (Figure 4a,b, \#\# $p<0.01$ ). 

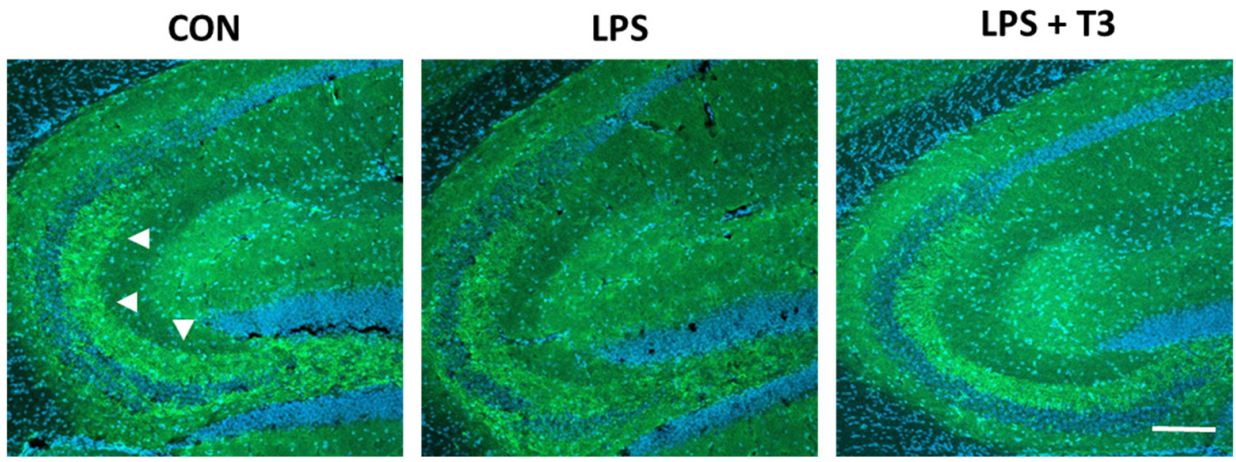

(a)

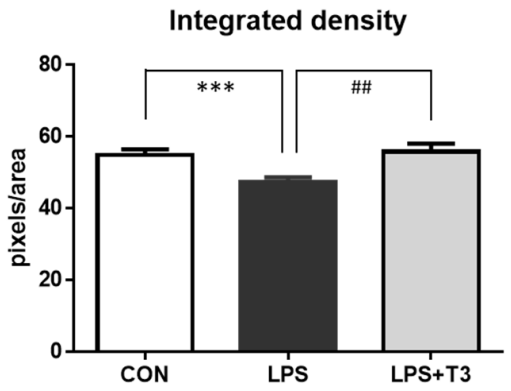

(b)

Figure 4. Effects of tocotrienols on synaptophysin immunoreactivity in the hippocampus in Experiment 2. (a) Sagittal sections were stained with the anti-synaptophysin antibody. Green signals show synaptophysin-positive signals, and typical signals were shown with white arrowheads. Nuclei were stained with DAPI (blue) (b) Quantification of synaptophysin-positive signals in the hippocampal CA3 stratum lucidum. Values are means \pm SEM $(n=24$, each group). Symbols show significant differences between CON vs. LPS (*** $p<0.001$; $t$-test) and LPS vs. LPS + T3 (\#\# $p<0.01$; $t$-test).

\subsection{HPLC Analysis of the Composition of Tocotrienols}

The composition of the tocotrienol mixture used in the present study was examined by an HPLC analysis (Figure 5). The quantification of each compound was calculated by preparing a calibration curve of the peak separated by HPLC using the corresponding standard. The results obtained showed that the mixture $(200 \mathrm{mg})$ comprised $\alpha$-tocotrienol (55.8 mg), $\beta$-tocotrienol (5.4 mg), $\gamma$-tocotrienol (92.6 mg), and $\delta$-tocotrienol (13.9 $\mathrm{mg}$ ).

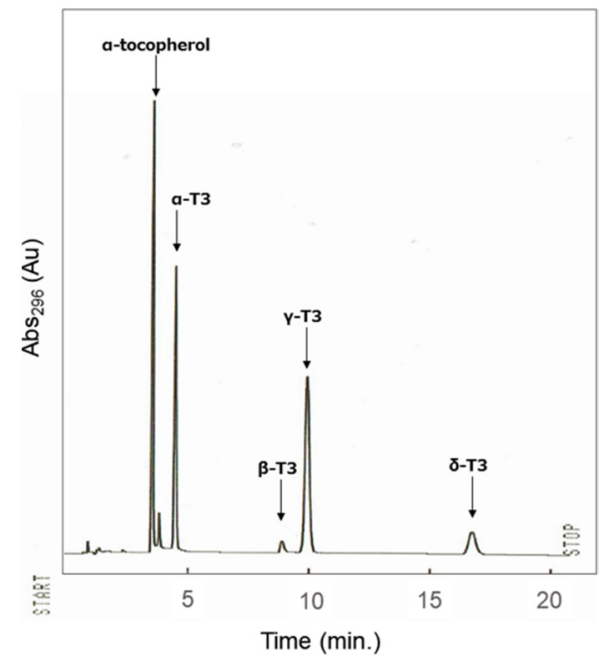

Figure 5. Chromatogram of the tocotrienol mixture used in the present study. 


\section{Discussion}

Chronic inflammation in peripheral tissue has been implicated in the pathogenesis of many diseases; therefore, the suppression of inflammatory responses by components in the daily diet is important. Chronic inflammation has been shown to affect brain function, and exogenous inflammation from peripheral tissue has a negative impact on the brain through the BBB $[4,5]$. In an aging society, the suppression of chronic inflammation is a key approach for maintaining a high quality of life. The effects of many anti-inflammatory compounds from natural sources have been examined using LPS-induced inflammation models [6-8]. In vitro and in vivo studies demonstrated that tocotrienols exhibited strong antioxidant and anti-inflammatory activities in peripheral tissue $[9,11]$. Tocotrienols have also been suggested to exert neuroprotective effects in several animal models, such as Alzheimer's disease and stroke [13,14]; however, in vivo research on the relationship between tocotrienols and inflammation in the brain has been limited.

We employed a tocotrienol mixture comprising four subtypes of tocotrienols in the present study. $\gamma$-Tocotrienol was the most abundant and accounted for more than one half of the total amount, while $\alpha$-tocotrienol comprised approximately one third. An in vitro study reported that $\alpha-, \gamma-$, and $\delta$-tocotrienols exerted anti-inflammatory effects on microglia [19]. In the present study, the tocotrienol mixture was p.o. administrated to mice and followed by an i.p. injection of LPS. For the duration of the administering of the tocotrienol mixture, no significant differences were observed in BW changes among the three groups examined. BW following the LPS injection were lower than in the CON group on Day 10 in experiment 1, but recovered in both the LPS and LPS + T3 groups by Day 15 in experiment 2.

In the immunohistochemical analysis of the brain hippocampus, microglia were significantly activated in the LPS group, with the accumulation of ameboid-type microglia. In contrast, microglial accumulation was significantly inhibited in the T3-treated group. Since microglial activation plays a role in inflammatory responses in the brain, we examined the hippocampal expression of HMGB1, a marker for delayed-onset inflammation and a non-infectious pro-inflammatory substance [20]. HMGB1 is released from cells in the brain after an inflammatory stimulation, binds to TLR4, and alters the signaling pathway of inflammation, such as nuclear factor kappa B, without any direct bacterial infection including LPS [21]. In the present study, the expression of HMGB1 was significantly up-regulated in the LPS group; however, it was not significantly down-regulated in the LPS + T3 group. It currently remains unclear whether tocotrienol is sufficiently potent to suppress the expression of HMGB1 in the brain based on the results obtained two days after the LPS injection.

In this animal model, immunocompetent cells in the blood, including monocytes, neutrophils, and lymphocytes, are activated by an LPS stimulation in peripheral tissue. These activated cells may cross the BBB because the penetration of LPS directly into the brain is unlikely. Therefore, microglial activation in the brain may be induced by the stimulation of immunocompetent cells [4,5]. Previous studies demonstrated that microglial activation induced neuronal cell damage in the brain [22]. In the present study, the expression of synaptophysin, a marker for synaptic vesicle membrane proteins, was observed in the CA3 stratum lucidum of the hippocampus in the CON group; however, its expression was significantly down-regulated in the LPS group. In contrast, immunopositive signals were maintained in the LPS + T3 group.

We propose two mechanisms of action by which the administration of the tocotrienol mixture to this mouse model suppressed microglial activation and maintained neuronal function in the hippocampus following LPS-induced inflammation. Unsaturated chains in the structure of tocotrienols have been suggested to enable their permeation into the brain [15]; therefore, the effects of suppression of microglial activation may be attributed to the direct functions of tocotrienols in the central nervous system. The tocotrienol mixture may also exert direct effects to protect neurons [23]. The other possibility is the suppression of inflammatory responses in peripheral tissue, namely, immunocompetent 
cells were inhibited peripherally by the anti-inflammatory effects of tocotrienols; therefore, un-activated immunocompetent cells did not spread inflammation to the brain.

The present results show the anti-inflammatory effects of tocotrienols in an in vivo model. The most remarkable point of the experiment was that, although the body weight decreased after LPS injection, and then was recovered after 7 days, the influence of inflammation on the synaptic condition in the brain remained at the same timepoint. Against this situation, even regarding small effects, tocotrienols showed a protective effect. The relationships between chronic inflammation and many diseases are a cause for concern in the current aging society, and, thus, it is important to suppress inflammation using components in the daily diet. The antioxidant effects of tocotrienols were previously shown to be more potent than those of tocopherol [15,24]; however, the sources of tocotrienols are limited, i.e., palm oil, rice bran, barley, wheat germ, and rye; therefore, the selective breeding of plants that are rich in tocotrienols is expected in the future. Neuroprotective effects of $\alpha$-tocotrienol have been examined in vitro [14]. To date, methods for precise analysis of tocotrienols have been reported, but methods for efficient sorting on a gram scale have not been established. For this reason, we are developing a new method for sorting tocotrienols on a gram scale and plan to apply it to the continuous development of this research. Though we used a tocotrienol mixture in the present study, comparison of the effect of each tocotrienol type, including $\alpha$-tocotrienol, will be clarified in the next study with this in vivo model.

Author Contributions: S.O. designed and performed the research and wrote the manuscript; M.A. performed the research and wrote the manuscript; M.M., Y.O., S.M., T.O., A.O., T.Y. and T.A. performed the research; A.S., M.N. and Y.F. provided research advice. All authors have read and agreed to the published version of the manuscript.

Funding: Some of this work was supported by The Collaborative Research and Education project between Ehime University and Matsuyama University.

Institutional Review Board Statement: All animal experiments were performed in accordance with the Guidelines for Animal Experimentation, approved by the Animal Care and Use Committee, and implemented using the approved protocol (\#17-011; 1 April 2018).

Conflicts of Interest: The authors declare no conflict of interest.

\section{References}

1. Liu, B.; Hong, J.S. Role of microglia in inflammation-mediated neurodegenerative diseases: Mechanisms and strategies for therapeutic intervention. J. Pharmacol. Exp. Ther. 2003, 304, 1-7. [CrossRef]

2. Qin, L.; Wu, X.; Block, M.L.; Liu, Y.; Breese, G.R.; Hong, J.S.; Knapp, D.J; Crews, F.T. Systemic LPS causes chronic neuroinflammation and progressive neurodegeneration. Glia 2007, 55, 453-462. [CrossRef] [PubMed]

3. Noh, H.; Jeon, J.; Seo, H. Systemic injection of LPS induces region-specific neuroinflammation and mitochondrial dysfunction in normal mouse brain. Neurochem. Int. 2014, 69, 35-40. [CrossRef] [PubMed]

4. Patel, J.P.; Frey, B.N. Disruption in the blood-brain barrier: The missing link between brain and body inflammation in bipolar disorder? Neural Plast. 2015, 2015, 708306. [CrossRef] [PubMed]

5. Fuggle, N.R.; Howe, F.A.; Allen, R.L.; Sofat, N. New insights into the impact of neuro-inflammation in rheumatoid arthritis. Front. Neurosci. 2014, 8, 357. [CrossRef] [PubMed]

6. Okuyama, S.; Yamamoto, K.; Mori, H.; Toyoda, N.; Yoshimura, M.; Amakura, Y.; Yoshida, T.; Sugawara, K.; Sudo, M.; Nakajima, M.; et al. Auraptene in the peels of Citrus kawachiensis (Kawachi Bankan) ameliorates lipopolysaccharide-induced inflammation in the mouse brain. Evid. Based Complement. Alternat. Med. 2014, 2014, 408503. [CrossRef] [PubMed]

7. Okuyama, S.; Makihata, N.; Yoshimura, M.; Amakura, Y.; Yoshida, T.; Nakajima, M.; Furukawa, Y. Oenothein B suppresses lipopolysaccharide (LPS)-induced inflammation in the mouse brain. Int. J. Mol. Sci. 2013, 14, 9767-9778. [CrossRef] [PubMed]

8. Haramiishi, R.; Okuyama, S.; Yoshimura, M.; Nakajima, M.; Furukawa, Y.; Ito, H.; Amakura, Y. Identification of the characteristic components in walnut and anti-inflammatory effect of glansreginin A as an indicator for quality evaluation. Biosci. Biotechnol. Biochem. 2020, 84, 187-197.

9. Ahsan, H.; Ahad, A.; Iqbal, J.; Siddiqui, W.A. Pharmacological potential of tocotrienols: A review. Nutr. Metab. 2014, 11, 52. [CrossRef]

10. Qureshi, A.A.; Reis, J.C.; Papasian, C.J.; Morrison, D.C.; Qureshi, N. Tocotrienols inhibit lipopolysaccharide-induced proinflammatory cytokines in macrophages of female mice. Lipids Health Dis. 2010, 9, 143. [CrossRef] 
11. Sen, C.K.; Khanna, S.; Roy, S. Tocotrienol: The natural vitamin E to defend the nervous system? Ann. N. Y. Acad. Sci. 2004, 1031, 127-142. [CrossRef]

12. Tan, S.W.; Abdullah, M.; Ali, D.A.I.; Vidyadaran, S. Palm tocotrienols reduce lipopolysaccharide-stimulated inflammatory responses of microglia. Malays. J. Med. Health Sci. 2016, 12, 1-8.

13. Chin, K.Y.; Tay, S.S. A review on the relationship between tocotrienol and alzheimer disease. Nutrients 2018, 10, 881. [CrossRef]

14. Khanna, S.; Roy, S.; Slivka, A.; Craft, T.K.; Chaki, S.; Rink, C.; Notestine, M.A.; DeVries, A.C.; Parinandi, N.L.; Sen, C.K. Neuroprotective properties of the natural vitamin E alpha-tocotrienol. Stroke 2005, 36, 2258-2264. [CrossRef]

15. Suzuki, Y.J.; Tsuchiya, M.; Wassall, S.R.; Choo, Y.M.; Govil, G.; Kagan, V.E.; Packer, L. Structural and dynamic membrane properties of alpha-tocopherol and alpha-tocotrienol: Implication to the molecular mechanism of their antioxidant potency. Biochemistry 1993, 32, 10692-10699. [CrossRef]

16. Panfili, G.; Fratianni, A.; Irano, M. Normal phase high-performance liquid chromatography method for the determination of tocopherols and tocotrienols in cereals. J. Agric. Food Chem. 2003, 51, 3940-3944. [CrossRef]

17. Shin, T.S.; Godber, J. Improved high-performance liquid chromatography of vitamin E vitamers on normal-phase columns. J. Am. Oil Chem. Soc. 1993, 70, 1289-1291. [CrossRef]

18. Podda, M.; Weber, C.; Traber, M.G.; Packer, L. Simultaneous determination of tissue tocopherols, tocotrienols, ubiquinols, and ubiquinones. J. Lipid Res. 1996, 37, 893-901. [CrossRef]

19. Tan, S.W.; Ramasamy, R.; Abdullah, M.; Vidyadaran, S. Inhibitory effects of palm alpha-, gamma- and delta-tocotrienol on lipopolysaccharide-induced nitric oxide production in BV2 microglia. Cell Immunol. 2011, 271, 205-209. [CrossRef]

20. Wang, H.; Yang, H.; Czura, C.J.; Sama, A.E.; Tracey, K.J. HMGB1 as a late mediator of lethal systemic inflammation. Am. J. Respir. Crit. Care Med. 2001, 164, 1768-1773. [CrossRef]

21. Mu, S.W.; Dang, Y.; Wang, S.S.; Gu, J.J. The role of high mobility group box 1 protein in acute cerebrovascular diseases. Biomed. Rep. 2018, 9, 191-197. [CrossRef]

22. Paudel, Y.N.; Angelopoulou, E.; Piperi, C.; Othman, I.; Shaikh, M.F. HMGB1-mediated neuroinflammatory responses in brain injuries: Potential mechanisms and therapeutic opportunities. Int. J. Mol. Sci. 2020, 21, 4609. [CrossRef]

23. Park, H.A.; Mnatsakanyan, N.; Broman, K.; Davis, A.U.; May, J.; Licznerski, P.; Crowe-White, K.M.; Lackey, K.H.; Jonas, E.A. Alpha-Tocotrienol Prevents Oxidative Stress-Mediated Post-Translational Cleavage of Bcl-xL in Primary Hippocampal Neurons. Int. J. Mol. Sci. 2019, 21, 220. [CrossRef]

24. Serbinova, E.A.; Packer, L. Antioxidant properties of alpha-tocopherol and alpha-tocotrienol. Methods Enzymol. 1994, 234, 354-366. 